\title{
Ultrasound-guided interventions with augmented reality in situ visualisation: a proof-of-mechanism phantom study
}

\author{
Nadja A. Farshad-Amacker ${ }^{1 *}$ (D) Till Bay ${ }^{2}$, Andrea B. Rosskopf ${ }^{1}$, José M. Spirig ${ }^{3}$, Florian Wanivenhaus ${ }^{3}$, \\ Christian W. A. Pfirrmann ${ }^{1}$ and Mazda Farshad ${ }^{3}$
}

\begin{abstract}
Background: Ultrasound (US) images are currently displayed on monitors, and their understanding needs good orientation skills. Direct overlay of US images onto the according anatomy is possible with augmented reality (AR) technologies. Our purpose was to explore the performance of US-guided needle placement with and without AR in situ US viewing.
\end{abstract}

Methods: Three untrained operators and two experienced radiologists performed 200 US-guided punctures: 100 with and 100 without AR in situ US. The punctures were performed in two different phantoms, a leg phantom with soft tissue lesions and a vessel phantom. Time to puncture and number of needle passes were recorded for each puncture. Data are reported as median [range] according to their non-normal distribution.

Results: AR in situ US resulted in reduced time (median [range], $13 \mathrm{~s}$ [3-101] versus 14 s [3-220]) and number of needle passes (median [range], 1 [1-4] versus 1 [1-8]) compared to the conventional technique. The initial gap in performance of untrained versus experienced operators with the conventional US (time, $21.5 \mathrm{~s}$ [3-220] versus $10.5 \mathrm{~s}$ [3-94] and needle passes 1 [1-8] versus $1[1,2])$ was reduced to $12.5 \mathrm{~s}$ [3-101] versus $13 \mathrm{~s}[3-100]$ and 1 [1-4] versus 1 [1-4] when using AR in situ US, respectively.

Conclusion: AR in situ US could be a potential breakthrough in US applications by simplifying operator's spatial orientation and reducing experience-based differences in performance of US-guided interventions. Further studies are needed to confirm these preliminary phantom results.

Keywords: Augmented reality, Holography, Phantoms (imaging), Punctures, Ultrasonography

\section{Key points}

- A novel augmented reality (AR) in situ ultrasound (US) technique has been proposed.

- AR technology allows for a direct overlay of US images onto the according anatomy.

- Using AR in situ US, operators could be faster and need less needle passes for US-guided interventions.

- AR in situ US may reduce experience-based differences in performing US-guided interventions.

\footnotetext{
* Correspondence: nadja.farshad@balgrist.ch

${ }^{1}$ Radiology, Balgrist University Hospital, Forchstrasse, 340, 8008 Zurich,

Switzerland

Full list of author information is available at the end of the article
}

\section{Background}

Although ultrasound (US) technology is radiation free and cost-effective, it is subject to experience dependency and highly dependent on the level of training $[1,2]$. In some institutions, diagnostic and therapeutic joint injections are performed under fluoroscopic guidance [3-5], even if US guidance for most joints would be possible [6, 7]. The main believed reason is reduction of observer dependency [8], paying a trade-off in terms of radiation exposure. Further fluoroscopic guidance usually is performed by injecting iodined contrast media for control of needle positioning, with the associated risk of allergic reactions [9]. Thus, a radiation-free modality such as US, but with a higher user-reproducibility, is desired. 
Augmented reality (AR) is increasingly gaining impact in medicine, particularly for guidance purposes since its introduction in the 1980s [10-12]. Some studies were recently published highlighting the use of AR in puncture guidance [10, 11, 13-17]. With AR headsets, computed visual information (holograms) are displayed in real time as an overlay of the user's field of view of reality.

We investigated if US with AR technology would achieve an AR in situ US view with the US image as hologram, displayed at the exact anatomic location in reality, creating a direct sonographic view for the operator. We hypothesised that AR in situ US would decrease interoperator variability in performance, by simplifying the challenge of spatial orientation.

\section{Methods}

No institutional review board was needed for this prospective phantom study.

\section{AR in situ US}

The AR in situ US system is composed of a conventional US system (SuperSonic Aixplorer Ultimate, SuperSonic Imagine, Aix-en-Provence, France), custom developed software, industry-grade head-mounted AR displays (Microsoft Hololens, Redmond, Washington, USA) and physical extensions of the US transducer handles (Fig. 1).

The positioning of the US image is based on calculating a relative offset of the image to the tracking marker on the US probe with respect to the head-mounted device coordinate system. To perform the offset calculation, a calibration station was used with an exact cut-out for the probe head and whose geometrical dimensions are precisely known. The station also has a tracking marker, which is recognised by the head-mounted device. To properly register, the US image is placed relative to the station marker according to the known offset to the cut-out. From this positioning, the offset to the probe's tracker marker is measured within the head-mounted device's coordinate system.

By observing the tracking pattern attached to the US transducer, the system calculates the correct positioning of the US image in space. The same tracking pattern is used to first calibrate the system together with the interpupillary distance of the operator's eyes. Using these coordinate system transformations and the information attained in the calibration step, the US image is transferred form the conventional US monitor to the AR headset. Thus, this transferred image is directly superimposed to the imaged anatomical structures. While scanning with the AR in situ US, the displayed superimposed image is update in real time as the operator moves the transducer, displaying the anatomical structures at their correct anatomical location in a 1:1 scale.

Additionally, to the superimposed imaged displayed in a 1:1 scale, the AR system also displays an enlarged version of the same image in an overhead position in the virtual threedimensional space. This allows the operator to consult both the superimposed 1:1 image at the anatomical location, as well as the same, but larger, image when looking up.

The accurate image size is calibrated based on the scanning depth and width given by the external US system. Changes in any of these two parameters make recalibration of our system necessary. Usually, this information is readily available from the US devices. The US image is displayed on a two-dimensional plane with the correct scan depth and width. The plane is then rendered onto the head-mounted device display and is scaled, positioned, and rotated in such a way that it is perceived at the correct three-dimensional location. For example, for in situ visualisation, the plane is shown beneath the US probe where the image is generated.

\section{Phantom puncture}

Three untrained operators (orthopaedic surgeons) without any experience in US and two experienced radiologists

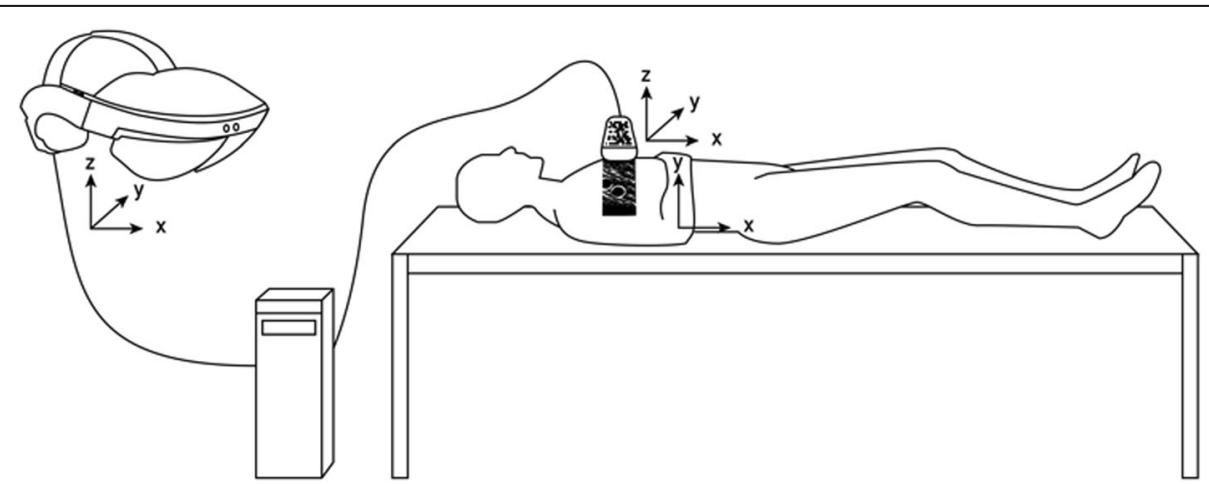

Fig. 1 AR in situ US system with the AR headset, the computer, where the spatial mapping of the US image is calculated by the custom software and the transducer showing its physically attached tracking pattern. Not shown here: the standard US that is providing the US image data to the software on the computer. AR Augmented reality, US Ultrasound 
(with 9 and 11 years of training) performed 200 USguided punctures with a linear transducer using a conventional US (SL 18-5, SuperSonic Aixplorer, SuperSonic Imagine, France) and with the AR in situ US (Microsoft Hololens and SuperSonic Aixplorer, SuperSonic Imagine, Aix-en-Provence, France).

The punctures were performed using a 20-gauge yellow needle (7-cm long) in a leg phantom (leg model with soft tissue biopsy insert, blue phantom, CAE Healthcare, Synmedic AG, Zurich, Switzerland) including 20 soft tissue lesions of varying lesions ranging from 4 to $11 \mathrm{~mm}$ in diameter. The leg phantom size was $81 \times 20 \times 20 \mathrm{~cm}$; the weight $11.5 \mathrm{~kg}$. Ten of those lesions were marked and numbered, ensuring that each operator punctured the same lesions in the same order (Figs. 2 and 3a). Further, each operator had to puncture the same vessel $(6 \mathrm{~mm}$ in size) in a vessel phantom (Blue phantom, CAE Healthcare, Synmedic AG, Zurich, Switzerland) ten times (Fig. 3b). These punctures were initially performed using AR in situ US, and $>4$ weeks later, each operator repeated the punctures using the conventional US, in order to avoid a possible training effect after the first puncture session. The

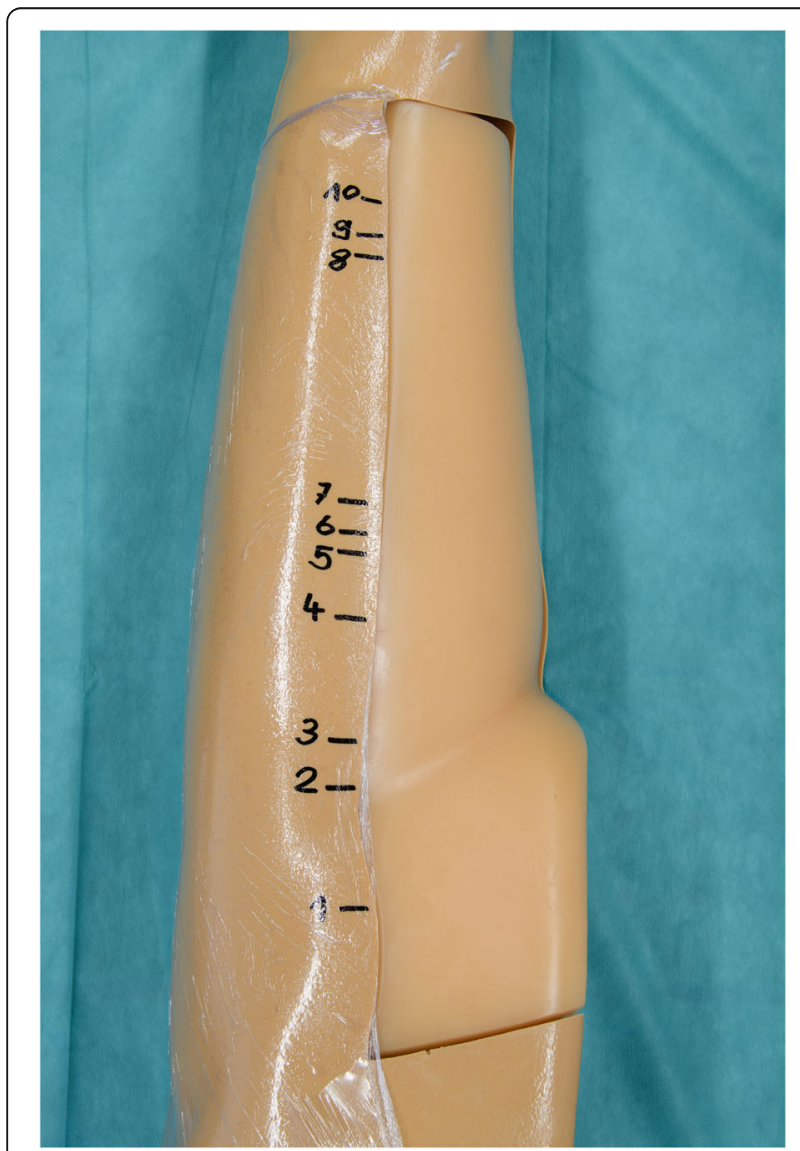

Fig. 2 The prepared leg phantom. Ten out of 20 lesions were marked, so that each operator punctuated the same lesions in the same order location of the needle tip was documented after each puncture by an experienced radiologist in US in the leg phantom and verified by fluid aspiration in the vessel phantom. Time to puncture and number of needle passes as well as location (correct or incorrect) of needle tips were documented for each puncture using the Redcap software (Vanderbilt University Medical Center, Version 8.11.5, Nashville, USA) [18].

\section{Data presentation}

Descriptive statistic was performed using the software PRISM (Version 8, Graphpad software, La Jolla (CA), USA). Medians and ranges were used to report the nonparametric data. By purpose, being this a proof-of mechanism phantom study for which sample size was not preliminarily estimated, and based on consultation with the statistician, we did not perform statistical testing for significance.

\section{Results \\ Conventional US versus AR in situ US}

The time to puncture was overall reduced using AR in situ US (13 s [3-101], median [range]) compared to the conventional US technique (14 s [3-220] (Fig. 4). The number of needle passes were also reduced using AR in situ US (1 [1-4], median [range]) compared to conventional US (1 [1-8], Fig. 5). Achieving correct location eventually was similar $(0.6[0.2-1.5]$ odds ratio $[95 \%$ confidence interval]) using either technique (AR in situ US, 90\% (90/10); conventional US, 94\% (94/6)).

\section{Inter-operator performances}

Experienced radiologists were faster and needed less needle passes when performing the punctures with the conventional US (time $10.5 \mathrm{~s}$ [3-94]; needle passes, $1[1,2]$ ) compared to the untrained operators (time 21.5 s [3-220]; needle passes, 1 [1-8]) (Fig. 6). When using AR in situ US, the difference between experienced and untrained operators was smaller (time $13 \mathrm{~s}$ [3-100] versus $12.5 \mathrm{~s}$ [3-101]; needle passes, 1 [1-4] versus 1 [1-4]; Fig. 6). The differences between untrained operators versus radiologists were more pronounced in the leg phantom. The differences of time and counted needle passes to puncture the targets within the different phantom types are summarised in Table 1.

\section{Discussion}

Ultrasound-guided punctures might be challenging and require training and expertise to be reliable and reproducible. We investigated if the combination of US with AR technology achieving an AR in situ US view with the US image as hologram, displayed at the exact anatomic location in reality, would reduce inter-operator variability in performance, by simplifying the challenge of 


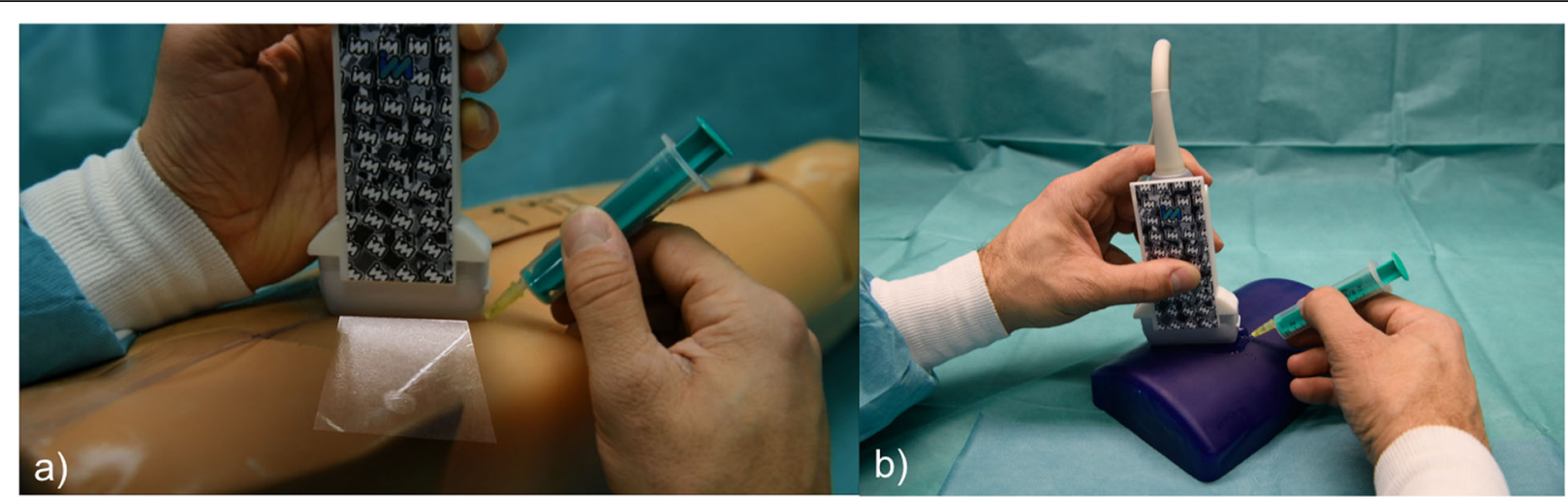

Fig. 3 Demonstration of the study setup, using augmented reality in situ ultrasound to puncture the soft tissue lesions in the leg phantom with operator view (a) or the vessel in the blue phantom without operator view (b)

spatial orientation. As expected, we observed a relevant difference in performance of untrained versus experienced operators using conventional US-guided punctures. This difference decreased when enforcing untrained operators with AR in situ US technology. The operator dependency is taught to be caused by differences in ability for spatial orientation. We assume that AR in situ US simplifies spatial orientation for US-guided interventions, since the US image is directly superposed on the anatomy in a 1:1 ratio and therefore the operator can directly aim the tip of the needle into the US image.

Not only the operator dependent differences were diminished, but also intraindividual differences, such as AR in situ US saved time and needle passes thorough all operators. We think this can simply be explained as the time turning the head to the monitor's image can be spared. Therefore, it is plausible that untrained operators needing more corrections also needed to turn their head more often to the monitor and thus needing more time in total compared to

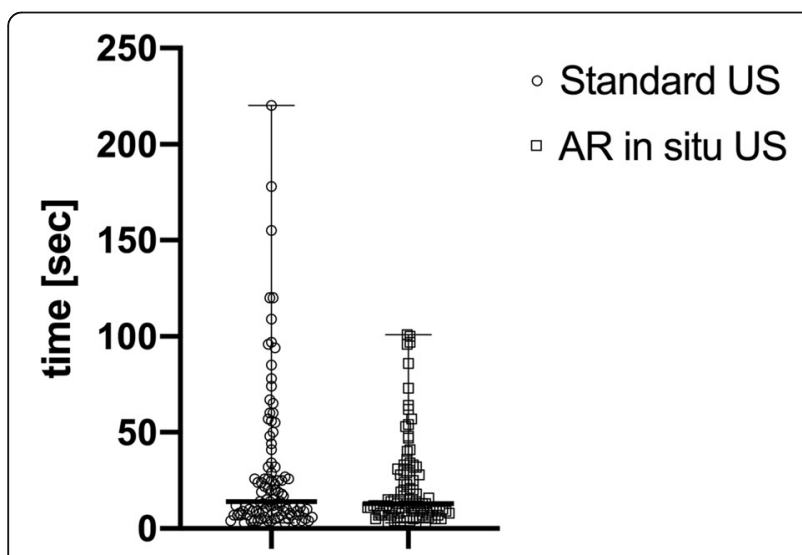

Fig. 4 Differences of time to target puncture of all operators using the standard ultrasound versus augmented reality in situ ultrasound technique the radiologists. The differences between untrained operators versus radiologists were more pronounced in the leg phantom, as the punctured targets were more difficult to puncture-the targets to puncture were in different depth within the soft tissue and deeper below the surface compared to the vessel phantom, where the blood vessel was a few centimetres below the surface and was for all punctures at the same height.

There are only few comparable US studies published using a similar technique with tracking the US probe and overlying the US image to its anatomical position [19, 20]. The study of Rosenthal et al. [19] found that operators performed better using the AR in situ technology; however, only needle placement accuracy were compared between the two methods (AR US versus standard US). Maas et al. [20] used the same technique to visualise the foetus on a portable device for the parents. Other studies using AR image overlay techniques for punctures needed manual alignment of the hologram, which was the main disadvantage [10]. The manual alignment is no longer needed with the here introduced method, as the image position is directly displayed at the anatomical position, that further increases precision of punctures, as malalignment of holograms or malalignments because of patient motions between image acquisition and puncture is negligible, in contrast to other studies [10,14, 21, 22].

Other advantages here not investigated could be the direct view to the patient at all times with AR in situ US, which could be important in case of patient motion, particularly considering other potential application such as breast mass biopsies, liver biopsies, and also injections for magnetic resonance arthrography or therapeutic joint injections. One other advantage of the use of AR in situ US using a head-mounted device is the better mobility and easier handling of the entire system. Further, even if we investigated feasibility with a linear probe, other probes (independent of the manufacturer) could be easily adaptable to the here presented technology. Also, the cost of head- 


\section{Standard US}

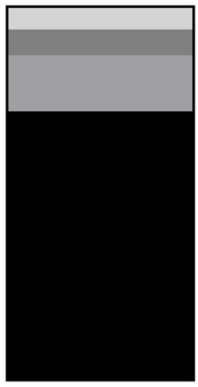

Total $=\mathbf{1 0 0}$
AR in situ US

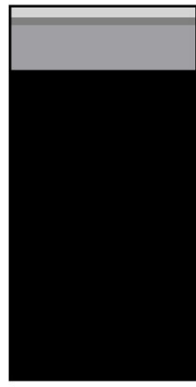

1 needle pass

2 needle passes

$\square 3$ needle passes

$\square>3$ needle passes

\section{Total $=\mathbf{1 0 0}$}

Fig. 5 Differences of number of needle passes to target puncture of all operators using the standard ultrasound versus augmented reality in situ ultrasound technique

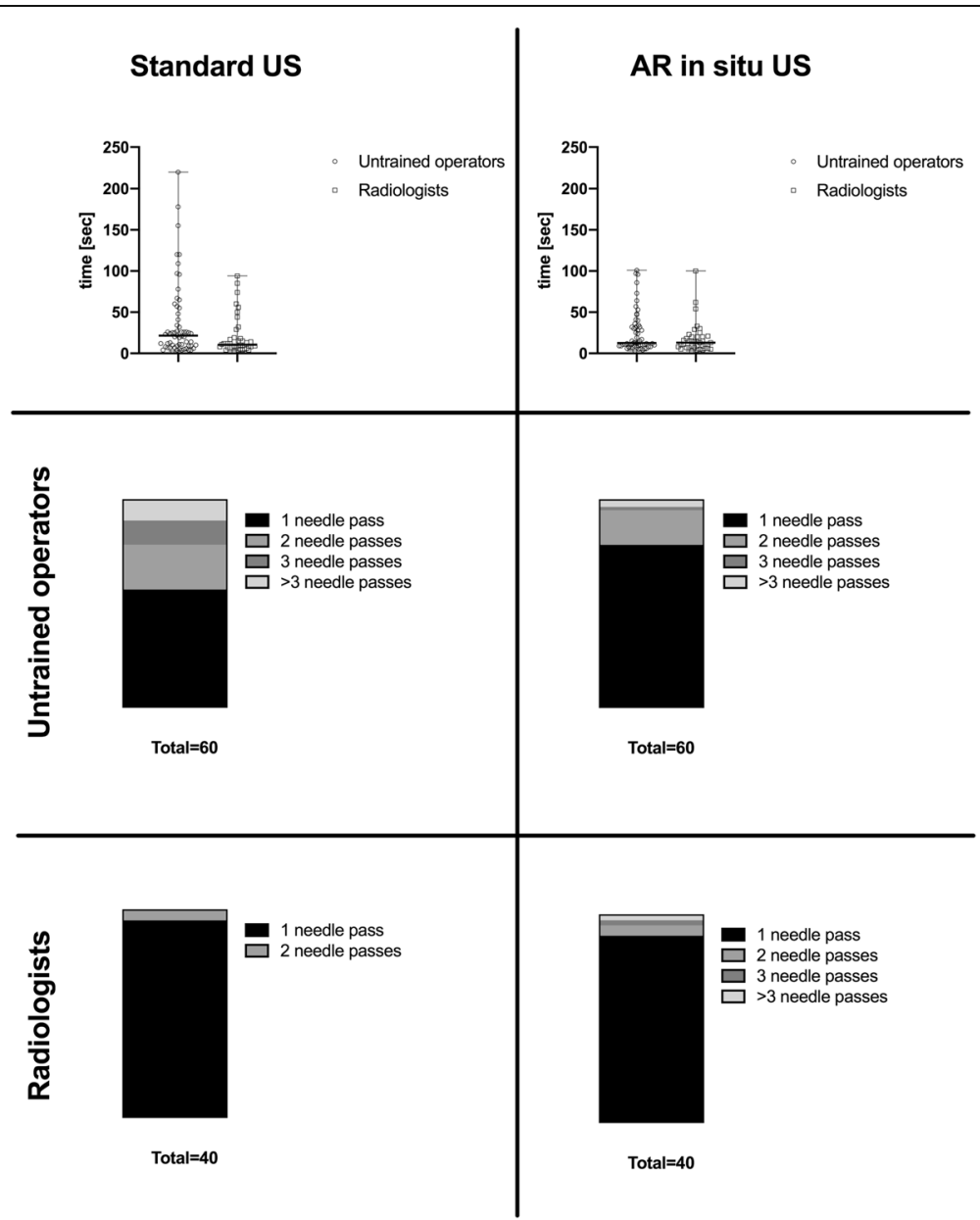

Fig. 6 Experience-based differences using the two different modalities (standard ultrasound versus augmented reality in situ ultrasound technique). Differences in time and number of needle passes to target puncture in untrained operators versus radiologists using standard ultrasound versus augmented reality in situ ultrasound technique 
Table 1 Time and counted needle passes using standard ultrasound versus augmented reality in situ ultrasound in the leg phantom and vessel phantom in untrained and trained operators

\begin{tabular}{|c|c|c|c|c|c|c|c|c|}
\hline & \multicolumn{2}{|c|}{$\begin{array}{l}\text { Standard US } \\
\text { leg phantom }\end{array}$} & \multicolumn{2}{|c|}{$\begin{array}{l}\text { Standard US } \\
\text { vessel phantom }\end{array}$} & \multicolumn{2}{|c|}{$\begin{array}{l}\text { AR in situ US } \\
\text { leg phantom }\end{array}$} & \multicolumn{2}{|c|}{$\begin{array}{l}\text { AR in situ US } \\
\text { vessel phantom }\end{array}$} \\
\hline & Untrained & Trained & Untrained & Trained & Untrained & Trained & Untrained & Trained \\
\hline Time (s), median [range] & $33[7-220]$ & $18.5[8-94]$ & $10.5[3-60]$ & $7[3-15]$ & $32[7-101]$ & $16.5[5-100]$ & 9 [3-34] & $9[3-25]$ \\
\hline Counted needle passes, median [range] & $2[1-8]$ & $1[1-2]$ & $1[1-6]$ & $1[1-1]$ & $1[1-4]$ & $1[1-4]$ & $1[1-2]$ & $1[1-1]$ \\
\hline
\end{tabular}

mounted devices has decreased over the last decades rapidly, making a potential cost-effectiveness of such system realistic in future.

Since this is the first study describing this potential breakthrough in US imaging, comparable study methodologies were difficult to identify. Two different phantoms, namely a leg phantom with soft tissue lesions and a phantom with vessels, were used to simulate two common clinical applications. Certainly, this is one of the main limitations of the study. External validity for clinical situations is not claimed and is subject of current investigations at our institution. We identified a further limitation that was difficult to address in designing this phantom study: repetitive interventions allow a learning curve and could have biased the measured outcomes. Therefore, first, the initial punctures were all performed using AR in situ US; on a separate day ( $>4$ weeks later), the same punctures were repeated using the conventional US. With this, we tried to reduce the difference of operator-dependent performance as a result of a potential learning curve.

In conclusion, this proof-of mechanism phantom study showed that AR in situ US technology could be a potential breakthrough for US-guided interventions by simplifying the operator's spatial orientation, with a potential for reducing the inter- and intra-operator variability.

\section{Abbreviations}

AR: Augmented reality; US: Ultrasound

\section{Acknowledgements}

We thank Tobias Götschi for his statistical consultation.

\section{Authors' contributions}

NF contributed to the study idea and design, preparing and performing of the experiments, and drafting and editing of the manuscript. TB contributed to the study idea and design, preparing of the experiments, and editing of the manuscript. AR contributed to the performing of the experiments and editing of the manuscript. IS contributed to the performing of the experiments and editing of the manuscript. FW contributed to the performing of the experiments and editing of the manuscript. CP contributed to supervising and editing of the manuscript. MF contributed to the study idea and design, supervising, preparing and performing of the experiments, and editing of the manuscript. All authors read and approved the final manuscript.

\section{Funding}

There was no industrial funding.
Consent for publication

Not applicable.

\section{Competing interests}

The authors declare a conflict of interest of TB and MF, who are involved in a 'start-up' company of the Balgrist University Hospital, which has developed the here presented software.

NF is married to MF.

\section{Author details}

${ }^{1}$ Radiology, Balgrist University Hospital, Forchstrasse, 340, 8008 Zurich, Switzerland. ${ }^{2}$ Incremed AG, Lenghalde 5, 8008 Zurich, Switzerland. ${ }^{3}$ Department of Orthopaedics, Balgrist University Hospital, University of Zurich, Forchstrasse 340, 8008 Zurich, Switzerland.

Received: 1 August 2019 Accepted: 29 October 2019

Published online: 04 February 2020

\section{References}

1. Nayahangan $\sqcup$, Nielsen KR, Albrecht-Beste E et al (2018) Determining procedures for simulation-based training in radiology: a nationwide needs assessment. Eur Radiol 28:2319-2327. https://doi.org/10.1007/s00330-017-5244-7

2. Kahr Rasmussen N, Andersen TT, Carlsen J et al (2019) Simulation-based training of ultrasound-guided procedures in radiology - a systematic review. Ultraschall Med. 40:584-602. https://doi.org/10.1055/a-0896-2714

3. Steinbach LS, Palmer WE, Schweitzer ME (2002) Special focus session. MR arthrography. Radiographics. 22:1223-1246. https://doi.org/10.1148/ radiographics.22.5.g02se301223

4. Ng AW, Hung EH, Griffith JF, Tong CS, Cho CC (2013) Comparison of ultrasound versus fluorcoscopic guided rotator cuff interval approach for MR arthrography. Clin Imaging 37:548-553. https://doi.org/10.1016/j.clinimag.2012.08.002

5. Messina C, Banfi G, Aliprandi A et al (2015) Ultrasound guidance to perform intra-articular injection of gadolinium-based contrast material for magnetic resonance arthrography as an alternative to fluoroscopy: the time is now. Eur Radiol 26:1221-1225. https://doi.org/10.1007/s00330-015-3945-3

6. Kantarci F, Ozbayrak M, Gulsen F, Gencturk M, Botanlioglu H, Mihmanli I (2013) Ultrasound-guided injection for MR arthrography of the hip: comparison of two different techniques. Skelet Radiol 42:37-42. https://doi. org/10.1007/s00256-011-1306-0

7. Souza PM, de Aguiar RO, Marchiori E, Bardoe SA (2010) Arthrography of the shoulder: a modified ultrasound guided technique of joint injection at the rotator interval. Eur J Radiol 74:e29-e32. https://doi.org/10.1016/j.ejrad.2009. 03.020

8. Lopez-Ben R, Coumas J (2016) Fluoroscopy versus sonography for image guidance in peripheral MSK intervention. Skeletal Radiol 45:1721-1722. https://doi.org/10.1007/s00256-016-2496-2

9. Hodler J (2008) Technical errors in MR arthrography. Skeletal Radiol 37:9-18. https://doi.org/10.1007/s00256-007-0329-z

10. Agten CA, Dennler C, Rosskopf AB, Jaberg L, Pfirrmann CWA, Farshad M (2018) Augmented reality-guided lumbar facet joint injections. Invest Radiol 53:495-498. https://doi.org/10.1097/RLI.0000000000000478

11. Yeo CT, Ungi T, U-Thainual P, Lasso A, McGraw RC, Fichtinger G (2011) The effect of augmented reality training on percutaneous needle placement in spinal facet joint injections. IEEE Trans Biomed Eng. 58(7):2031-7. https://doi. org/10.1109/TBME.2011.2132131.

12. Bosc R, Fitoussi A, Hersant B, Dao TH, Meningaud JP (2019) Intraoperative augmented reality with heads-up displays in maxillofacial surgery: a systematic review of the literature and a classification of relevant 
technologies. Int J Oral Maxillofac Surg 48:132-139. https://doi.org/10.1016/j. ijom.2018.09.010

13. Fritz J, U-Thainual P, Ungi T et al (2012) Augmented reality visualisation using an image overlay system for MR-guided interventions: technical performance of spine injection procedures in human cadavers at 1.5 tesla. Eur Radiol 23:235-245. https://doi.org/10.1007/s00330-012-2569-0

14. Fritz J, U-Thainual P, Ungi T et al (2013) Augmented reality visualization using image overlay technology for MR-guided interventions: cadaveric bone biopsy at 1.5 T. Invest Radiol 48:464-470. https://doi.org/10.1097/RLI. 0b013e31827b9f86

15. Pratt $P$, Ives M, Lawton $G$ et al (2018) Through the HoloLens ${ }^{\mathrm{TM}}$ looking glass: augmented reality for extremity reconstruction surgery using $3 \mathrm{D}$ vascular models with perforating vessels. Eur Radiol Exp 2:2. https://doi.org/ 10.1186/s41747-017-0033-2

16. Solbiati M, Passera KM, Rotilio A et al (2018) Augmented reality for interventional oncology: proof-of-concept study of a novel high-end guidance system platform. Eur Radiol Exp 2:18. https://doi.org/10.1186/ s41747-018-0054-5

17. Fritz J, U-Thainual P, Ungi T et al (2012) Augmented reality visualization with image overlay for MRI-guided intervention: accuracy for lumbar spinal procedures with a 1.5-T MRI system. AJR Am J Roentgenol 198:W266-W273. https://doi.org/10.2214/AJR.11.6918

18. Harris PA, Taylor R, Thielke R, Payne J, Gonzalez N, Conde JG (2009) Research electronic data capture (REDCap)--a metadata-driven methodology and workflow process for providing translational research informatics support. J Biomed Inform 42:377-381. https://doi.org/10.1016/j.jbi.2008.08.010

19. Rosenthal M, State A, Lee J et al (2002) Augmented reality guidance for needle biopsies: an initial randomized, controlled trial in phantoms. Med Image Anal 6:313-320. https://doi.org/10.1016/S1361-8415(02)00088-9

20. Maas S, Sobotta C, Ingler M (2016) Study on augmented reality 4-D ultrasound in prenatal medicine. Ultraschall Med 37:PS9_14. https://doi.org/ 10.1055/s-0036-1587918

21. Weiss CR, Marker DR, Fischer GS, Fichtinger G, Machado AJ, Carrino JA (2012) Augmented reality visualization using image-overlay for MR-guided interventions: system description, feasibility, and initial evaluation in a spine phantom. AJR Am J Roentgenol 196:W305-W307. https://doi.org/10.2214/ AJR.10.5038

22. Fritz J, U-Thainual P, Ungi T et al (2012) Augmented reality visualization with use of image overlay technology for MR imaging-guided interventions: assessment of performance in cadaveric shoulder and hip arthrography at 1.5 T. Radiology 265:254-259. https://doi.org/10.1148/radiol.12112640

\section{Publisher's Note}

Springer Nature remains neutral with regard to jurisdictional claims in published maps and institutional affiliations.

\section{Submit your manuscript to a SpringerOpen ${ }^{\circ}$ journal and benefit from:}

- Convenient online submission

- Rigorous peer review

- Open access: articles freely available online

- High visibility within the field

- Retaining the copyright to your article

Submit your next manuscript at $\boldsymbol{\nabla}$ springeropen.com 